\title{
Effect of Weight Loss on Survival in Esophageal Cancer Patients undergoing Neoadjuvant Chemoradiotherapy and Surgery
}

\author{
Jia Hua Lyu ${ }^{1,2}$, Tao Li ${ }^{1,2}$, Yong Tao Han ${ }^{1,3}$, Lei Wu ${ }^{2}$, Lin Peng ${ }^{3}$, Qi Feng Wang ${ }^{2}$, Long Liang ${ }^{2}$ \\ ${ }^{1}$ Department of the Chest Radiation Oncology, School of Medicine, University of Electronic Science and Technology of China, \\ Sichuan, Chengdu, Sichuan 610041, China; ${ }^{2}$ Department of Radiotherapy, Sichuan Cancer Hospital \& Institute, School of Clinical \\ Medicine, University of Electronic Science and Technology of China, Chengdu 610041, Sichuan, China; ${ }^{3}$ Department of Thoracic \\ Surgery, Sichuan Cancer Hospital \& Institute, Chengdu, Sichuan 610041, Sichuan, China
}

\begin{abstract}
Objective This study aimed to determine the impact of weight loss during neoadjuvant chemoradiotherapy on the survival of patients with esophageal carcinoma. Methods We retrospectively examined 102 consecutive patients with esophageal carcinoma who underwent neoadjuvant chemoradiotherapy followed by radical resection at Sichuan Cancer Hospital \& Institute between 2003 and 2017. The patients were divided into three groups based on the amount of body weight lost during neoadjuvant chemoradiotherapy: severe weight loss $(>10 \%)$, high weight loss $(5 \% \sim 10 \%)$, and low weight loss $(<5 \%)$. The correlations of weight loss with toxicity, progressionfree survival, and overall survival were investigated. Results The median overall survival was 49.7 months in the low weight loss group compared with 35.4 and 25.1 months in the high and severe weight loss groups $(P=0.041)$. The 1-year overall survival rates in the severe, high, and low weight loss groups were $62.5 \%, 85.0 \%$, and $90.7 \%$, respectively; the corresponding 3-year overall survival rates were $21.9 \%, 47.3 \%$, and $68.8 \%$, respectively, and the corresponding 5 -year overall survival rates were $21.9 \%, 31.0 \%$, and $44.4 \%$, respectively. The multivariate analysis indicated that a pathological complete response and severe weight loss were independent prognostic factors for overall survival. Any leukopenia $(P=0.024)$, leukopenia of at least grade $3(P=0.021)$, and anemia $(P=0.042)$ occurred more frequently in the severe weight loss group. Conclusions Weight loss during neoadjuvant CRT is an independent and adverse prognostic factor in esophageal carcinoma patients, whereas a stable weight confers a better prognosis.
\end{abstract}

Key words: Esophageal cancer; Prognosis; Weight loss; Neoadjuvant chemoradiotherapy; Toxicity

\section{Introduction}

Neoadjuvant chemoradiotherapy (CRT) followed by surgery has been established as an effective treatment for patients with locally advanced esophageal cancer. Several well-designed randomized controlled trials have demonstrated that neoadjuvant CRT improved outcome measures compared with surgery alone, including the $\mathrm{R} 0$ resection, pathological complete response (pCR), locoregional control, and survival rates [1-3]. However, the overall survival (OS) varies in such patients, and some fail to benefit from neoadjuvant chemoradiotherapy, indicating a need to identify the factors associated with the prognosis and provide more information about the most appropriate clinical interventions to improve outcomes among highrisk patients. One such factor is weight loss, which is common and often occurs by the time of diagnosis or during treatment in patients with esophageal cancer [4-8]. Although weight loss before treatment has been shown to be a key prognostic indicator of the OS in such patients [9-

Corresponding author: Tao Li, MD, PhD, Department of Radiotherapy, Sichuan Cancer Hospital \& Institute, School of Clinical Medicine, University of Electronic Science and Technology of China, Chengdu 610041, Sichuan, China; Tel:+86 289542 0173; Fax: +86 288542 0590; Email: litaoxmf@126.com
12], the impact of weight loss during neoadjuvant CRT on the long-term survival of patients with esophageal cancer remains unknown. Therefore, this retrospective study aimed to investigate the association of weight loss during neoadjuvant CRT with survival outcomes in patients with esophageal cancer.

\section{Materials \& Methods \\ Study design and participants}

A total of 102 patients with esophageal squamous cell cancer undergoing neoadjuvant CRT followed by surgery at Sichuan Cancer Hospital \& Institute from March 2003 to August 2017 were retrospectively identified. Enrolled patients met the following inclusion criteria: (1) newly diagnosed, histologically-confirmed primary esophageal squamous cell cancer; (2) clinically stage II or III before treatment according to the 2002 American Joint Committee on Cancer Staging System (version 6.0); (3) Karnofsky performance score $\geq 80$; and (4) patients underwent neoadjuvant chemoradiotherapy followed by radical esophagectomy. The exclusion criteria were as follows: (1) missing weight records pre- or post-CRT; (2) metastatic disease before or during preoperative treatment; (3) nonsquamous cell carcinoma; and (4) other active malignancy (except for basal cell carcinoma of the skin). The patient 
demographics, neoadjuvant treatment, toxicity, weight records and follow-up results were reviewed in detail from the medical records and the follow-up system. The current study was undertaken in accordance with the ethical standards of the World Medical Association Declaration of Helsinki. A waiver of informed consent was requested, and this study was approved by Sichuan Cancer Hospital \& Institute.

The patients were divided into three groups based on the extent of body weight lost during neoadjuvant chemoradiotherapy: severe weight loss (>10\%; SWL group), high weight loss (5\% 10\%; HWL group), and low weight loss $(<5 \%$; LWL group).

\section{Neoadjuvant chemoradiotherapy}

All patients underwent neoadjuvant CRT and surgery. The external beam radiotherapy was performed with a $6 \sim 10$ MV linear accelerator. Irradiation was scheduled to deliver a total dose of 36 40 Gy to the primary tumor and any positive regional lymph nodes in fractions of 2 3 Gy daily on five consecutive days per week for $2 \sim 4$ weeks. One of two dual-agent chemotherapy regimens was used (either cisplatin plus 5-fluorouracil or cisplatin plus paclitaxel) and was administered every three weeks in two cycles, concurrent with the radiotherapy. All patients underwent radical esophagectomy $3 \sim 8$ weeks after the completion of preoperative radiotherapy.

\section{Surgery}

Surgery was carried out after a median of 6 weeks ( $3 \sim 8$ weeks) of rest after neoadjuvant CRT. The primary surgical procedure in each case was determined by an experienced medical group. Ivor Lewis esophagogastrectomy or McKeown esophagogastrectomy was selected according to tumor location and lesion length.

\section{Measurements and follow-up}

Patient's weights were measured at the initial visit and weekly thereafter. Weight loss during neoadjuvant treatment was defined as the difference between the baseline weight and the final weight recorded after completing all neoadjuvant treatment. The OS was measured from the first day of treatment to the day of mortality or last follow-up, if the patient was still alive. The progressionfree survival (PFS) was defined as the time between the initiation of treatment and disease progression or mortality from any cause. A pCR was defined as follows: the absence of viable tumor cells with only fibrotic masses or cellular mucin pools present in the area of the primary tumor and lymph nodes. Acute toxicities occurring during CRT were graded according to the Common Terminology Criteria for Adverse Events (CTCAE, version 4.0) and the Radiation Therapy Oncology Group criteria. After surgery, follow-up evaluations were conducted every 3 6 months for the first
2 years and every $6 \sim 12$ months thereafter. Data regarding the medical history, physical examination, blood tests, endoscopy results, and computed tomography scans of the neck, chest, or abdomen were collected.

\section{Statistical analysis}

Analyses were performed using IBM SPSS Version 20 (SPSS Statistics V20, IBM Corporation, New York, USA). Continuous variables are presented as the means (standard deviation, SD) or as medians (95\% confidence interval) and were compared using Student's $t$-tests. Categorical variables were compared between groups with chi-squared or Fisher's exact tests, as appropriate. Survival curves were generated using the Kaplan-Meier method, and differences between groups were assessed by the log-rank test. Multivariate Cox proportional hazards models were used to identify independent prognostic factors for OS, with only those variables that were statistically significant in univariate Cox models being included. All statistical tests were two-sided and the significance level was set at 0.05 .

\section{Results \\ Patient characteristics}

The data for 102 patients with esophageal squamous cell cancer who had undergone neoadjuvant CRT followed by surgery during the study period were analyzed. The overall weight loss during neoadjuvant CRT ranged from $-8.89 \%$ to $16.67 \%$ (median: $5.2 \%$ ).

To investigate the effects of weight loss on the prognosis, patients were categorized into three subgroups based on their weight loss during neoadjuvant treatment. The SWL group comprised 16 patients (15.7\%), the HWL group comprised 42 patients $(41.2 \%)$, and the LWL group comprised 44 patients $(43.1 \%)$. There were no significant differences in the gender, age, cTN stage, Karnofsky performance score, lesion length, primary lesion site, or chemotherapy regimen among the subgroups (Table 1). In addition, there were no significant differences between the three groups in terms of the pathological outcomes after neoadjuvant CRT, including the ypT stage, ypN stage, $\mathrm{pCR}$, and pathologic downstaging rates.

\section{Toxicity due to CRT}

Regarding the toxicity associated with neoadjuvant CRT, we found that the complication rates tended to be comparable among the groups. However, any grade leukopenia $(P=0.024)$, leukopenia of at least grade $3(P=$ $0.021)$, and anemia $(P=0.042)$ occurred more frequently in the SWL group (Table 2).

\section{Survival outcomes}

The median follow-up duration across the whole study population was 33 months. A Kaplan-Meier analysis of the OS showed a significant difference among the groups. 
Table 1 Clinical and pathological characteristics of patients.

\begin{tabular}{|c|c|c|c|c|}
\hline Variables & LWL group & HWL group & SWL group & $P$ \\
\hline \multicolumn{5}{|l|}{ Clinical variables } \\
\hline Age, years & & & & 0.756 \\
\hline$\leq 60$ & 29 & 28 & 9 & \\
\hline$>60$ & 15 & 14 & 7 & \\
\hline Sex & & & & 0.548 \\
\hline Male & 37 & 32 & 14 & \\
\hline Female & 7 & 10 & 2 & \\
\hline KPS score & & & & 0.687 \\
\hline $90-100$ & 27 & 22 & 10 & \\
\hline 80 & 17 & 20 & 6 & \\
\hline Length of the lesion, $\mathrm{cm}$ & & & & 0.563 \\
\hline$\leq 5$ & 16 & 19 & 5 & \\
\hline$>5$ & 28 & 23 & 11 & \\
\hline Primary lesion site & & & & 0.352 \\
\hline Upper & 12 & 7 & 1 & \\
\hline Middle & 24 & 26 & 13 & \\
\hline Lower & 8 & 9 & 2 & \\
\hline cT stage & & & & 0.523 \\
\hline 2 & 8 & 4 & 2 & \\
\hline 3 & 21 & 26 & 11 & \\
\hline 4 & 15 & 12 & 3 & \\
\hline cN stage & & & & 0.206 \\
\hline No & 22 & 13 & 6 & \\
\hline $\mathrm{N}+$ & 22 & 29 & 10 & \\
\hline Chemotherapy regimen & & & & 0.165 \\
\hline $\mathrm{PF}$ & 31 & 27 & 7 & \\
\hline $\mathrm{TP}$ & 13 & 15 & 9 & \\
\hline \multicolumn{5}{|l|}{ Pathological variables } \\
\hline ypT stage & & & & 0.379 \\
\hline 0 & 20 & 13 & 5 & \\
\hline $1 \sim 2$ & 16 & 14 & 7 & \\
\hline $3 \sim 4$ & 8 & 15 & 4 & \\
\hline ypN stage & & & & 0.502 \\
\hline N0 & 29 & 24 & 8 & \\
\hline $\mathrm{N}+$ & 15 & 18 & 8 & \\
\hline Achievement of $\mathrm{pCR}$ & & & & 0.126 \\
\hline Yes & 19 & 10 & 4 & \\
\hline No & 25 & 32 & 12 & \\
\hline Pathological downstaging & & & & 0.141 \\
\hline Yes & 39 & 32 & 11 & \\
\hline No & 5 & 10 & 5 & \\
\hline
\end{tabular}

Abbreviations: KPS, Karnofsky Performance Score; pCR, pathological complete response; TP, cisplatin with paclitaxel; PF, cisplatin with 5-fluorouracil; LWL, low weight loss $(<5 \%$ of the body weight loss); HWL, high weight loss(loss of $5-10 \%$ of the body weight); SWL, sever weight loss (loss of $>10 \%$ of the body weight); cT stage, clinical tumor stage; cN stage, clinical node stage; ypT stage, pathological tumor stage after neoadjuvant chemoradiotherapy; ypN stage, pathological node stage after neoadjuvant chemoradiotherapy. 
The median OS was 25.1 months (95\% CI, 5.1 45.1) in the SWL group $(P=0.041), 35.4$ months $(95 \% \mathrm{CI}, 19.7 \sim 51.1)$ in the HWL group, and 49.7 months $(95 \%$ CI, 36.6 62.8) in the LWL group. The respective OS rates in the SWL, HWL, and LWL groups were $62.5 \%, 85.0 \%$, and $90.7 \%$ at 1 year; $21.9 \%, 47.3 \%$, and $68.8 \%$ at 3 years; and $21.9 \%, 31.0 \%$, and $44.4 \%$ at 5 years (Figure 1). The median progressionfree survival rates were 25.0 months (95\% CI, 11.6 38.4)

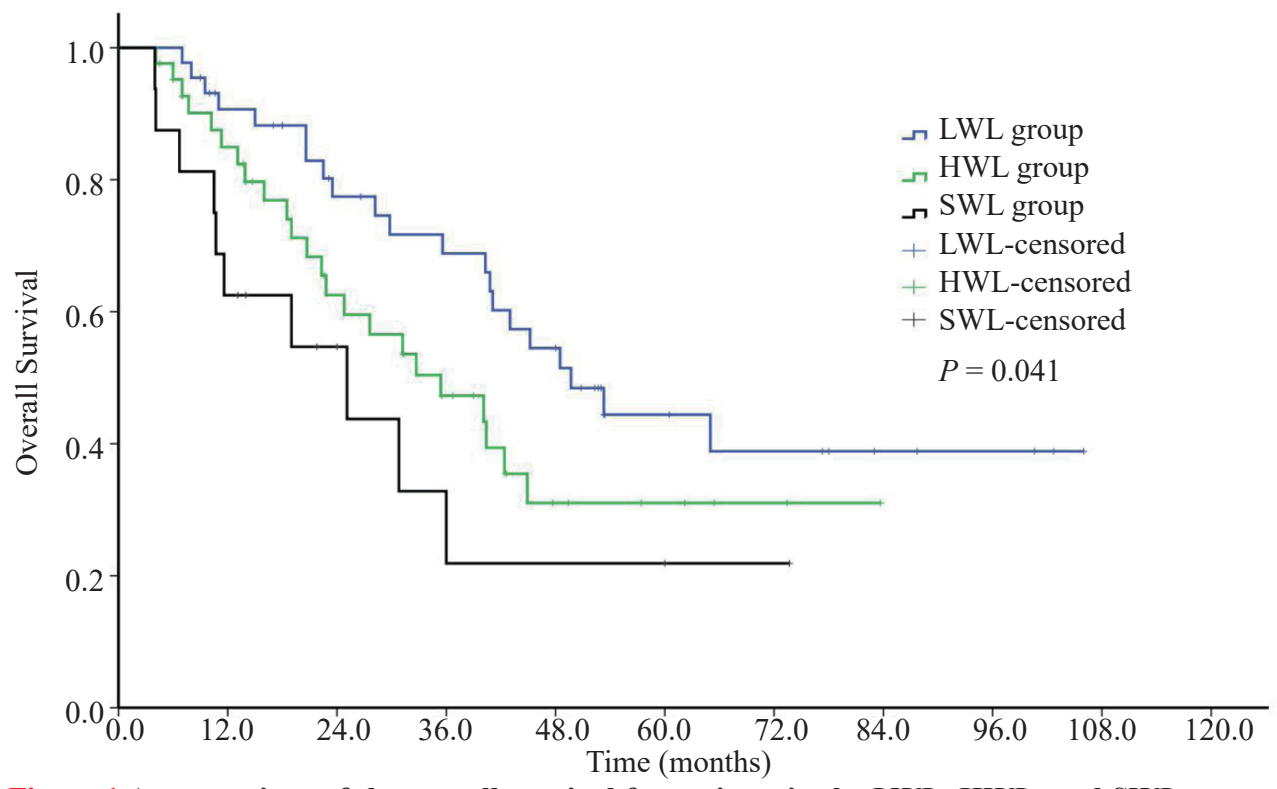

Figure 1 A comparison of the overall survival for patients in the LWL, HWL, and SWL groups. Abbreviations: LWL, low weight loss $(<5 \%$ body weight loss); HWL, high weight loss (loss of $5 \% \sim 10 \%$ of body weight); SWL, severe weight loss (loss of $>10 \%$ of body weight)

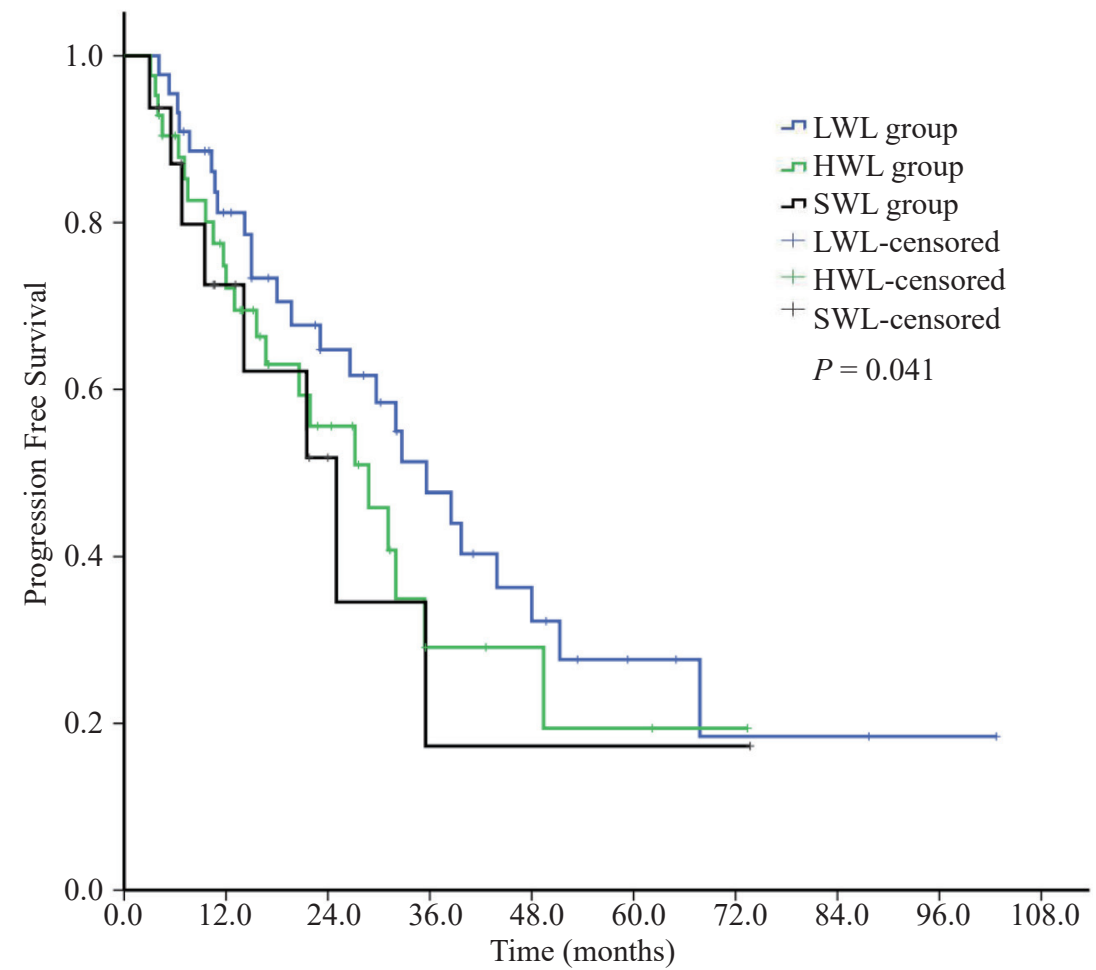

Figure 2 A comparison of the progression-free survival for patients in the LWL, HWL, and SWL groups. Abbreviations: LWL, low weight loss $(<5 \%$ body weight loss); HWL, high weight loss (loss of $5 \% \sim 10 \%$ of body weight); SWL, severe weight loss (loss of $>10 \%$ of body weight) 
in the SWL group, 28.8 months (95\% CI, 17.3 40.3) in the HWL group, and 35.6 months (95\% CI, 25.2 46.0) in the LWL group ( $P=0.411$; Figure 2$)$.

As shown in Table 3, the univariate analysis revealed that severe weight loss $(>10 \%)$ during neoadjuvant CRT and an advanced pathological $\mathrm{T}$ or $\mathrm{N}$ stage were associated with a poor OS, whereas achieving $\mathrm{pCR}$ and downstaging were associated with a better OS. The multivariate analysis subsequently indicated that achieving pCR and sustaining less weight loss during neoadjuvant CRT were independent predictors of a more favorable OS (Table 4).

\section{Discussion}

It is apparent that the survival of patients with esophageal cancer is determined by not only the tumor pathology, but also host-related factors, such as the nutritional status [13-17]. The patient's body weight, serum albumin and hemoglobin levels, and the Patient Generated Subjective Global Assessment score (PG-SGA) are the most commonly-used methods for evaluating the nutritional status. However, body weight measurement is perhaps the most crucial indicator, and is both inexpensive and easy to perform.

Indeed, unintentional weight loss is a major concern both before and during the treatment of cancer. Several studies have demonstrated that weight loss before esophageal cancer resection might be related to the postoperative survival [6-12]. To our knowledge, no research has focused on weight loss during neoadjuvant CRT or on the relationship between this weight loss and the survival outcomes in patients with esophageal cancer. In our study, the range of weight loss during neoadjuvant CRT was $-8.89 \%$ to $16.67 \%$ (median: $5.2 \%$ ), with $45.1 \%, 39.2 \%$, and $17 \%$ of patients experiencing LWL, HWL, and SWL, respectively. The Kaplan-Meier analysis revealed that the OS was significantly lower in the SWL group compared with the HWL and LWL groups $(P<0.05)$, whereas the multivariate analysis revealed that severe weight loss was a risk factor for a poor OS. Overall, the median OS was much higher in the LWL group (49.7 months) than in the HWL group (34.1 months); however, the difference was not significant $(P=0.105)$. This may due to the limited sample sizes, indicating the need for a larger sample in future research.

Although it remains unclear why weight loss affects the survival outcomes in patients with esophageal cancer, some hypotheses have been suggested. One potential reason is that weight loss during neoadjuvant CRT is accompanied by insufficient energy and nutrient intake that is required for body growth, tissue repair, disease recovery, normal physiological function, and immune function (especially cell-mediated immunity). A deficit in energy and nutrient intake may impair the nutritional and performance status, increase the risk of treatment-related toxicity, and impair the efficacy of therapy for esophageal cancer, especially when the weight loss is severe [18-20]. In addition, highly

Table 2 Toxicities during neo-adjuvant chemoradiotherapy.

\begin{tabular}{|c|c|c|c|c|}
\hline Toxicities & $\begin{array}{c}\text { LWL group } \\
n=44(\%)\end{array}$ & $\begin{array}{c}\text { HWL group } \\
\text { n=42(\%) }\end{array}$ & $\begin{array}{c}\text { SWL group } \\
\mathrm{n}=16(\%)\end{array}$ & $P$ \\
\hline \multicolumn{5}{|l|}{ Leukopenia } \\
\hline Any grade & $32(72.7)$ & $37(88.1)$ & $16(100.0)$ & 0.024 \\
\hline$\geq$ Grade 3 & $12(27.3)$ & $21(50.0)$ & $10(62.5)$ & 0.021 \\
\hline \multicolumn{5}{|l|}{ Anemia } \\
\hline Any grade & $20(45.5)$ & $24(57.1)$ & $13(81.3)$ & 0.042 \\
\hline$\geq$ Grade 3 & $3(6.8)$ & $2(4.8)$ & $2(12.5)$ & 0.511 \\
\hline \multicolumn{5}{|c|}{ Thrombocytopenia } \\
\hline Any grade & $7(15.9)$ & $11(26.2)$ & $3(18.8)$ & 0.541 \\
\hline$\geq$ Grade 3 & $0(0.0)$ & $3(7.1)$ & $1(6.3)$ & 0.164 \\
\hline \multicolumn{5}{|c|}{ Anorexia/ Vomiting } \\
\hline Any grade & $17(38.6)$ & $18(42.9)$ & $8(50.0)$ & 0.741 \\
\hline$\geq$ Grade 3 & $1(2.3)$ & $3(7.1)$ & $2(12.5)$ & 0.209 \\
\hline \multicolumn{5}{|c|}{ Radiation esophagitis } \\
\hline Any grade & $16(36.4)$ & $12(28.6)$ & $7(43.8)$ & 0.521 \\
\hline$\geq$ Grade 3 & $1(2.3)$ & $3(7.1)$ & $1(6.3)$ & 0.469 \\
\hline \multicolumn{5}{|c|}{ Radiation pneumonitis } \\
\hline Any grade & $7(15.9)$ & $7(16.7)$ & $3(18.8)$ & 1.000 \\
\hline$\geq$ Grade 3 & $1(2.3)$ & $0(0.0)$ & $1(6.3)$ & 0.290 \\
\hline
\end{tabular}

Abbreviations: LWL, low weight loss; HWL, high weight loss; SWL, sever weight loss 
Table 3 Results of the univariate analysis demonstrating factors associated with overall survival.

\begin{tabular}{|c|c|c|}
\hline Subgroup & HR $(95 \%$ CI) & $P$ \\
\hline \multicolumn{3}{|c|}{ Weight loss during neoadjuvant CRT } \\
\hline Low weight loss & 1.00 & \\
\hline High weight loss & $1.641(0.902 \sim 2.982)$ & 0.105 \\
\hline Severe weight loss & $2.519(1.175 \sim 5.402)$ & 0.018 \\
\hline \multicolumn{3}{|l|}{ Age, years } \\
\hline$\leq 60$ & 1.00 & \\
\hline$>60$ & $1.199(0.668 \sim 2.153)$ & 0.544 \\
\hline \multicolumn{3}{|l|}{ Sex } \\
\hline Male & 1.00 & \\
\hline Female & $1.255(0.660 \sim 2.386)$ & 0.488 \\
\hline \multicolumn{3}{|l|}{ KPS score } \\
\hline $90 \sim 100$ & 1.00 & \\
\hline 80 & $1.358(0.780 \sim 2.365)$ & 0.279 \\
\hline \multicolumn{3}{|c|}{ Length of the lesion, $\mathrm{cm}$} \\
\hline$\leq 5$ & 1.00 & \\
\hline$>5$ & $1.021(0.595 \sim 1.752)$ & 0.940 \\
\hline \multicolumn{3}{|l|}{ Tumor location } \\
\hline Upper & 1.00 & \\
\hline Middle & $0.619(0.326 \sim 1.177)$ & 0.144 \\
\hline Lower & $0.846(0.383 \sim 1.869)$ & 0.679 \\
\hline \multicolumn{3}{|l|}{ cT stage } \\
\hline 2 & 1.00 & \\
\hline 3 & $1.148(0.445 \sim 2.961)$ & 0.775 \\
\hline 4 & $2.059(0.763 \sim 5.556)$ & 0.154 \\
\hline \multicolumn{3}{|l|}{$\mathrm{cN}$ stage } \\
\hline N0 & 1.00 & \\
\hline $\mathrm{N}+$ & $1.423(0.818 \sim 2.476)$ & 0.212 \\
\hline \multicolumn{3}{|c|}{ Chemotherapy regimens } \\
\hline $\mathrm{PF}$ & 1.00 & \\
\hline ТP & $0.986(0.548 \sim 1.776)$ & 0.963 \\
\hline \multicolumn{3}{|l|}{ ypT stage } \\
\hline 0 & 1.00 & \\
\hline $1 \sim 2$ & $1.624(0.844 \sim 3.126)$ & 0.147 \\
\hline $3 \sim 4$ & 2.307 (1.179 4.513) & 0.015 \\
\hline \multicolumn{3}{|l|}{ ypN stage } \\
\hline N0 & 1.00 & \\
\hline $\mathrm{N}+$ & $2.119(1.233 \sim 3.642)$ & 0.007 \\
\hline \multicolumn{3}{|l|}{ Achievement of pCR } \\
\hline Yes & 1.00 & \\
\hline No & $2.367(1.284 \sim 4.362)$ & 0.006 \\
\hline \multicolumn{3}{|l|}{ Downstaging } \\
\hline Yes & 1.00 & \\
\hline No & $2.461(1.284 \sim 4.717)$ & 0.007 \\
\hline
\end{tabular}

Abbreviations: low weight loss $(<5 \%$ of the body weight loss); high weight loss (loss of $5 \% \sim 10 \%$ of the body weight); severe weight loss (loss of $>10 \%$ of the body weight); KPS, Karnofsky Performance Score; pCR, pathological complete response; TP, cisplatin with paclitaxel, PF, cisplatin with 5 fluorouracil; cT stage, clinical tumor stage; $\mathrm{cN}$ stage, clinical node stage; ypT stage, pathological tumor stage after neoadjuvant chemoradiotherapy; ypN stage, pathological node stage after neoadjuvant chemoradiotherapy. 
malignant tumors usually grow rapidly and have active metabolism, potentially leading to progressive body mass consumption and weight loss [21]. In this way, weight loss can be seen as a marker of a higher resting energy expenditure and more aggressive and rapid cancer proliferation, and as such, confers a worse prognosis.

A second potential reason for the association between weight loss and the OS is that greater weight loss during radiotherapy may cause positioning errors that reduce the accuracy and efficacy of radiotherapy. This has been demonstrated by $\mathrm{Wu} \mathrm{ZG}$ et al. [22], who reported that positioning errors during radiotherapy increased with weight loss in patients with thoracic and abdominal cancers.

Another finding of our study is that less extensive weight loss may be protective against myelosuppression. Patients who had severe weight loss also had a substantially increased incidence of leukopenia and anemia compared with patients who had less weight loss, possibly because patients with less weight loss had a better nutritional status, which may have improved the recovery time from CRTinduced myelosuppression. A decreased hemoglobin concentration can also reduce tissue oxygenation and the radiosensitivity of cancer cells, further influencing the response of patients to CRT [23-27].

Severe weight loss may cause tumor-associated cachexia in certain patients. This syndrome of skeletal muscle mass depletion occurs due to systemic inflammation, anorexia, and mechanical and neuroendocrine changes [28]. Cancer cachexia reflects a state of systemic inflammation in which elevations in various circulating inflammatory markers, such as serum C-reactive protein and interleukin-6, are associated with an increased risk of disease progression, tumor invasion, lymph node metastasis, and a poorer response to CRT [29-31]. When muscle loss causes greater physical burdens and disability, it is categorized as frailty and is associated with increased risks of all-cause mortality, postoperative mortality, and treatment-related complications [32].

Therefore, attention must be paid to the nutritional status of patients during anticancer therapy. Some prospective studies have demonstrated that nutritional support combined with anticancer therapy could prolong the survival in patients with head and neck cancer [33], gastric cancer [34], colorectal cancer [35], and esophageal cancer [36]. A pilot randomized controlled trial was performed in patients with locally advanced esophageal cancer undergoing neoadjuvant CRT, among whom 30 patients received a "walk-andeat intervention" (nurse-supervised walking plus weekly nutritional advice after medical nutrition therapy) and 29 patients underwent care as usual. During neoadjuvant CRT, the patients who received the walk-and-eat intervention lost less body weight $(-0.8 \mathrm{~kg}$ vs $-3.5 \mathrm{~kg}, P<0.001)$ compared to usual care group [37]. Nutritional interventions should be introduced early and tailored to each patient undergoing preoperative CRT for esophageal cancer. This should include a personalized weight and food intake evaluation,

Table 4 Results of the multivariate analysis demonstrating factors associated with overall survival.

\begin{tabular}{lcc}
\hline Subgroup & HR $\mathbf{( 9 5 \% ~ C I ) ~}$ & $\boldsymbol{P}$ \\
\hline Weight loss during neoaduvant CRT & 1.00 & 0.222 \\
$\quad$ Low weight loss & $1.476(0.790 \sim 2.759)$ & 0.008 \\
High weight loss & $2.911(1.320 \sim 6.419)$ & \\
Severe weight loss & & 0.286 \\
ypT stage & 1.00 & 0.951 \\
0 & $1.030(0.396 \sim 2.680)$ & \\
$1 \sim 2$ & $1.925(0.579 \sim 6.403)$ & 0.349 \\
$3 \sim 4$ & 1.00 & \\
ypN stage & & 0.027 \\
N0 & 1.00 & \\
N+ & $390(0.698 \sim 2.770)$ & \\
Achievement of pCR & & 0.327 \\
Yes & $1.686(0.593 \sim 4.796)$ & \\
No & & \\
Downstaging & & \\
Yes & & \\
No & & \\
\hline
\end{tabular}

Abbreviation: low weight loss $(<5 \%$ of the body weight loss); high weight loss (loss of $5 \% \sim 10 \%$ of the body weight); severe weight loss (loss of $>10 \%$ of the body weight); pCR, pathological complete response; ypT stage, pathological tumor stage after neoadjuvant chemoradiotherapy; ypN stage, pathological node stage after neoadjuvant chemoradiotherapy. 
dietary counseling, and supplemental nutrition support (if necessary).

Several potential limitations of the present study should be acknowledged. First, this study was retrospective, which might have introduced heterogeneity and bias. In addition, previous studies have reported that skeletal muscle and visceral adipose tissue loss might be the underlying factors contributing to poor survival [38-40]; however, we did not evaluate the impact of changes in body composition during preoperative CRT. Despite these limitations, our study has clarified the importance of changes in body weight and highlights the need for nutritional support during neoadjuvant treatment in patients with esophageal squamous cell cancer. This can form the basis for prospective clinical trials with larger sample sizes. These trials should probe further into the relationship between weight loss and the OS in patients with esophageal cancer who undergo neoadjuvant CRT before surgery.

In conclusion, weight loss during neoadjuvant CRT compromises the survival of patients with esophageal cancer. Routine and accurate monitoring of weight changes, together with patient education, nutritional counseling, and proper nutritional support during neoadjuvant treatment, should be considered integral to the multidisciplinary care of patients with locally advanced esophageal cancer.

\section{Future Perspective}

The relationship between body weight loss and the survival of esophageal cancer patients is becoming clearer. However, there are still many areas of controversy and many areas where information is lacking. The relationships among nutritional therapy, body composition alterations and survival, and the value and impact of specific nutrients such as 3-PUFAs on the body weight and anti-cancer treatment outcomes, requires more in-depth research. Fortunately, there has been an increasing number of research studies focusing on these topics. The results of these studies will certainly provide more updated and powerful evidence for the importance of the nutritional status and nutritional treatment with regard to the outcomes of EC patients.

\section{Executive Summary}

1) Although weight loss before treatment has been shown to be a key prognostic indicator of the OS in EC patients, the impact of weight loss during neoadjuvant CRT on the long-term survival of patients with esophageal cancer remains unknown.

2) A total of 102 patients who underwent neoadjuvant chemoradiotherapy followed by surgery were divided into three groups based on the extent of body weight lost during neoadjuvant chemoradiotherapy: severe weight loss (> $10 \%)$, high weight loss (5\% 10\%), and low weight loss (< $5 \%)$.

3) This study revealed that the OS was significantly lower in the severe weight loss group compared with the other groups.

4) A multivariate analysis indicated that a pCR and severe weight loss were independent prognostic factors for overall survival.

5) It remains unclear why weight loss affects the survival outcomes in patients with esophageal cancer. However, there are some hypotheses: (1) weight loss during neoadjuvant CRT is accompanied by insufficient energy and nutrient intake; (2) greater weight loss during radiotherapy may cause positioning errors which reduce the accuracy and efficacy of radiotherapy; (3) patients who had severe weight loss also had a substantially increased incidence of anemia, which reduced tissue oxygenation and therefore reduced the radiosensitivity of the tumor; (4) severe weight loss may cause tumor-associated cachexia and frailty, which are associated with increased risks of all-cause mortality.

6) Nutritional interventions should be introduced early and should be specifically targeted to each patient undergoing preoperative CRT for esophageal cancer.

\section{Author Contributions}

Jia Hua Lyu and Tao Li conceived the study, manuscript, and statistical analysis; Qi Feng Wang, Yong Tao Han, Long Liang, Lei $\mathrm{Wu}$, and Lin Peng provided assistance with collecting clinical data; all authors read and approved the final manuscript.

\section{Conflict of Interest}

None of the authors has any conflict of interest to disclose.

\section{Ethical disclosure}

The present study was approved by the Institutional Review Board of Sichuan Cancer Hospital \& Institute and was conducted in accordance with the guidelines of the International Conference on Harmonization/Good Clinical Practice and the principles of the Declaration of Helsinki.

\section{Fundings}

This work was supported by the Sichuan Province Health Research Project (No. 19PJ276)

\section{References}

1. Van HP, Hulshof MC, Van LJJ, Steyerberg EW, Van BHMI, Wijnhoven BP, Richel DJ, Nieuwenhuijzen GA, Hospers GA, Bonenkamp JJ, Cuesta MA, Blaisse RJ, Busch OR, Ten KFJ, Creemers GJ, Punt CJ, Plukker JT, Verheul HM, Spillenaar BEJ, Van DH, Van DSMJ, Rozema T, Biermann K, Beukema JC, Piet AH, Van RCM, Reinders JG, Tilanus HW, Van DGA. Preoperative chemoradiotherapy for esophageal or junctional cancer. N Engl J Med 2012;366(22):2074-84.

2. Yang H, Liu H, Chen Y, Zhu C, Fang W, Yu Z, Mao W, Xiang J, Han Y, Chen Z, Yang H, Wang J, Pang Q, Zheng X, Yang H, Li T, Lordick F, D'Journo XB, Cerfolio RJ, Korst RJ, Novoa NM, Swanson SJ, 
Brunelli A, Ismail M, Fernando HC, Zhang X, Li Q, Wang G, Chen B, Mao T, Kong M, Guo X, Lin T, Liu M, Fu J. Neoadjuvant chemoradiotherapy followed by surgery versus surgery alone for locally advanced squamous cell carcinoma of the esophagus (neocrtec5010): a phase iii multicenter, randomized, open-label clinical trial. J Clin Oncol 2018;36(27):2796-803.

3. Shapiro J, Van LJJB, Hulshof MCCM, Van HP, Van BHMI, Wijnhoven BPL, Van LHWM, Nieuwenhuijzen GAP, Hospers GAP, Bonenkamp JJ, Cuesta MA, Blaisse RJB, Busch ORC, Ten KFJW, Creemers GM, Punt CJA, Plukker JTM, Verheul HMW, Bilgen EJS, Van DH, Van DSMJC, Rozema T, Biermann K, Beukema JC, Piet AHM, Van RCM, Reinders JG, Tilanus HW, Steyerberg EW, Van DGA. Neoadjuvant chemoradiotherapy plus surgery versus surgery alone for oesophageal or junctional cancer (CROSS): long-term results of a randomised controlled trial. Lancet Oncol 2015;16(9):1090-8

4. Daly JM, Fry WA, Little AG, Winchester DP, McKee RF, Stewart AK, Fremgen AM. Esophageal cancer: results of an American College of Surgeons Patient Care Evaluation Study. J Am Coll Surg 2000;190(5):562-72.

5. Riccardi D, Allen K. Nutritional management of patients with esophageal and esophagogastric junction cancer. Cancer Control 1999;6(1):64-72.

6. Liu B, Cheng B, Wang C, Chen P, Cheng Y. The prognostic significance of metabolic syndrome and weight loss in esophageal squamous cell carcinoma. SCI REP 2018;8(1):10101.

7. Shen S, Araujo JL, Altorki NK, Sonett JR, Rodriguez A, Sungur-Stasik K, Spinelli CF, Neugut AI, Abrams JA. Variation by stage in the effects of prediagnosis weight loss on mortality in a prospective cohort of esophageal cancer patients. Dis Esophagus 2017;30(9):1-7.

8. Kitagawa H, Namikawa T, Munekage M, Fujisawa K, Munekage E, Kawanishi Y, Kobayashi M, Hanazaki K. Analysis of factors associated with weight loss after esophagectomy for esophageal cancer. Anticancer Res 2016;36(10):5409-12.

9. Van DSMK, Tilanus HW, Van LJJ, Johar AM, Lagergren P, Lagergren J, Wijnhoven BP. The influence of preoperative weight loss on the postoperative course after esophageal cancer resection. J Thorac Cardiovasc Surg 2014;147(1):490-5.

10. D'Journo XB, Ouattara M, Loundou A, Trousse D, Dahan L, Nathalie T, Doddoli C, Seitz JF, Thomas PA. Prognostic impact of weight loss in 1-year survivors after transthoracic esophagectomy for cancer. Dis Esophagus 2012;25(6):527-34.

11. Yu XL, Yang J, Chen T, Liu YM, Xue WP, Wang MH, Bai SM. Excessive pretreatment weight loss is a risk factor for the survival outcome of esophageal carcinoma patients undergoing radical surgery and postoperative adjuvant chemotherapy. Can J Gastroenterol Hepatol 2018;2018(6075207):8.

12. Martin L, Lagergren P. Risk factors for weight loss among patients surviving 5 years after esophageal cancer surgery. Ann Surg Oncol 2015;22(2):610-6.

13. Sakai M, Sohda M, Miyazaki T, Yoshida T, Kumakura Y, Honjo H, Hara K, Ozawa D, Suzuki S, Tanaka N, Yokobori T, Kuwano H. Association of preoperative nutritional status with prognosis in patients with esophageal cancer undergoing salvage esophagectomy. Anticancer Res 2018;38(2):933-8.

14. Rietveld SCM, Witvliet-van NJE, Ottens-Oussoren K, Van DPDL,
De Vdsmae. The prediction of deterioration of nutritional status during chemoradiation therapy in patients with esophageal cancer. Nutr Cancer 2018;70(2):229-35.

15. Song T, Wan Q, Yu W, Li J, Lu S, Xie C, Wang H, Fang M. Pretreatment nutritional risk scores and performance status are prognostic factors in esophageal cancer patients treated with definitive chemoradiotherapy. Oncotarget 2017;8(58):98974-84.

16. Mak M, Bell K, Ng W, Lee M. Nutritional status, management and clinical outcomes in patients with esophageal and gastro-oesophageal cancers: a descriptive study. Nutr Diet 2017;74(3):229-35.

17. Bollschweiler E, Herbold T, Plum P, Holscher AH. Prognostic relevance of nutritional status in patients with advanced esophageal cancer. Expert Rev Anticancer Ther 2013;13(3):275-8.

18. Sunpaweravong S, Puttawibul P, Ruangsin S, Laohawiriyakamol S, Sunpaweravong P, Sangthawan D, Pradutkanchana J, Raungkhajorn P, Geater A. Randomized study of antiinflammatory and immune-modulatory effects of enteral immunonutrition during concurrent chemoradiotherapy for esophageal cancer. Nutr Cancer 2014;66(1):1-5.

19. De APSJ, Emilia DSFM, Waitzberg DL. Omega-3 supplements for patients in chemotherapy and/or radiotherapy: a systematic review. Clin Nutr 2015;34(3):359-66.

20. Fontana L, Eagon JC, Colonna M, Klein S. Impaired mononuclear cell immune function in extreme obesity is corrected by weight loss. Rejuvenation Res 2007;10(1):41-6.

21. Cao DX, Wu GH, Zhang B, Quan YJ, Wei J, Jin H, Jiang Y, Yang ZA. Resting energy expenditure and body composition in patients with newly detected cancer. Clin Nutr 2010;29(1):72-7.

22. Wu ZG, Huang JW, Liu LB, Yang HS, Qu YY. Effect of body weight change on positioning error of chest and abdomen patients receiving IMRT. Fujian Medical Journal 2011;33(3):103-5.

23. Varlotto J, Stevenson MA. Anemia, tumor hypoxemia, and the cancer patient. Int J Radiat Oncol Biol Phys 2005;63(1):25-36.

24. Rades D, Schild SE, Bahrehmand R, Zschenker O, Alberti WA, Rudat VR. Prognostic factors in the nonsurgical treatment of esophageal carcinoma with radiotherapy or radiochemotherapy: the importance of pretreatment hemoglobin levels. Cancer 2005;103(8):1740-6

25. Zhang HQ, Wang RB, Yan HJ, Zhao W, Zhu KL, Jiang SM, Hu XG, Yu JM. Prognostic significance of CYFRA21-1, CEA and hemoglobin in patients with esophageal squamous cancer undergoing concurrent chemoradiotherapy. Asian Pac J Cancer Prev 2012;13(1):199-203.

26. Hamai Y, Hihara J, Taomoto J, Yamakita I, Ibuki Y, Okada M. Hemoglobin level influences tumor response and survival after neoadjuvant chemoradiotherapy for esophageal squamous cell carcinoma. World J Surg 2014;38(8):2046-51.

27. Kato Y, Yashiro M, Fuyuhiro Y, Kashiwagi S, Matsuoka J, Hirakawa T, Noda S, Aomatsu N, Hasegawa T, Matsuzaki T, Sawada T, Ohira M, Hirakawa K. Effects of acute and chronic hypoxia on the radiosensitivity of gastric and esophageal cancer cells. Anticancer Res 2011;31(10):3369-75.

28. Anandavadivelan P, Lagergren P. Cachexia in patients with oesophageal cancer. Nat Rev Clin Oncol 2016;13(3):185-98.

29. Huang Y, Feng JF, Liu JS, Chen QX. Prognostic role of serum C-reactive protein in esophageal cancer: a systematic review and meta-analysis. Ther Clin Risk Manag 2015;11(1):89-94.

30. Groblewska M, Mroczko B, Sosnowska D, Szmitkowski M. Inter- 
leukin 6 and C-reactive protein in esophageal cancer. Clin Chim Acta 2012;413(19-20):1583-90

31. Fujiwara H, Suchi K, Okamura S, Okamura H, Umehara S, Todo M, Shiozaki A, Kubota T, Ichikawa D, Okamoto K, Ochiai T, Kokuba Y, Sonoyama T, Otsuji E. Elevated serum CRP levels after induction chemoradiotherapy reflect poor treatment response in association with IL-6 in serum and local tumor site in patients with advanced esophageal cancer. J Surg Oncol 2011;103(1):62-8.

32. Brown JC, Harhay MO, Harhay MN. The prognostic importance of frailty in cancer survivors. J Am Geriatr Soc 2015;63(12):2538-43.

33. Muller-Richter U, Betz C, Hartmann S, Brands RC. Nutrition management for head and neck cancer patients improves clinical outcome and survival. Nutr Res 2017;48:1-8.

34. Qiu M, Zhou YX, Jin Y, Wang ZX, Wei XL, Han HY, Ye WF, Zhou ZW, Zhang DS, Wang FH, Li YH, Yang DJ, Xu RH. Nutrition support can bring survival benefit to high nutrition risk gastric cancer patients who received chemotherapy. Support Care Cancer 2015;23(7):1933-9.

35. Ravasco $P$, Monteiro-Grillo I, Camilo M. Individualized nutrition intervention is of major benefit to colorectal cancer patients: long-term follow-up of a randomized controlled trial of nutritional therapy. Am J Clin Nutr 2012;96(6):1346-53.
36. Cox S, Powell C, Carter B, Hurt C, Mukherjee S, Crosby TD. Role of nutritional status and intervention in oesophageal cancer treated with definitive chemoradiotherapy: outcomes from SCOPE1. Br J Cancer 2016;115(2):172-7.

37. Xu YJ, Cheng JC, Lee JM, Huang PM, Huang GH, Chen CC. A Walk-and-eat intervention improves outcomes for patients with esophageal cancer undergoing neoadjuvant chemoradiotherapy. Oncologist 2015;20(10):1216-22.

38. Dalal S, Hui D, Bidaut L, Lem K, Del Fabbro E, Crane C, Reyes-Gibby CC, Bedi D, Bruera E. Relationships among body mass index, longitudinal body composition alterations, and survival in patients with locally advanced pancreatic cancer receiving chemoradiation: a pilot study. J Pain Symptom Manage 2012;44(2):181-91.

39. Bowden JCS, Williams LJ, Simms A, Price A, Campbell S, Fallon MT, Fearon KCH. Prediction of 90 day and overall survival after chemoradiotherapy for lung cancer: role of performance status and body composition. Clin Oncol (R Coll Radiol) 2017;29(9):576-84.

40. Boshier PR, Heneghan R, Markar SR, Baracos VE, Low DE. Assessment of body composition and sarcopenia in patients with esophageal cancer: a systematic review and meta-analysis. Dis Esophagus 2018;31(8):1-11. 